\title{
In Vitro Interaction between Isavuconazole and Tacrolimus, Cyclosporin A, or Sirolimus against Aspergillus Species
}

\author{
Patrick Schwarz $1,2, *$ (D) and Eric Dannaoui ${ }^{3,4,5}$ (D) \\ 1 Department of Internal Medicine, Respiratory and Critical Care Medicine, University Hospital Marburg, \\ D-35043 Marburg, Germany \\ 2 Center for Invasive Mycoses and Antifungals, Philipps University Marburg, D-35037 Marburg, Germany \\ 3 Dynamyc Research Group (EA 7380), Faculté de Médecine de Créteil, \\ Université Paris-Est-Créteil-Val-de-Marne, F-94010 Créteil, France; eric.dannaoui@aphp.fr \\ 4 Unité de Parasitologie-Mycologie, Hôpital Européen Georges-Pompidou, F-75015 Paris, France \\ 5 Faculté de Médecine, Université de Paris, 75006 Paris, France \\ * Correspondence: patrick.schwarz@med.uni-marburg.de; Tel.: +49-6421-5862464
}

Received: 17 May 2020; Accepted: 16 June 2020; Published: 8 July 2020

\begin{abstract}
The interaction of isavuconazole with immunosuppressors (tacrolimus, cyclosporin A, or sirolimus) against 30 Aspergillus isolates belonging to the most common species responsible for invasive aspergillosis in humans (Aspergillus flavus, Aspergillus fumigatus, Aspergillus nidulans, Aspergillus niger, and Aspergillus terreus) was evaluated in vitro by a microdilution checkerboard technique based on the EUCAST reference method for antifungal susceptibility testing. The interpretation of the results was performed based on the fractional inhibitory concentration index. The combination of isavuconazole with tacrolimus, cyclosporin A, or sirolimus, was synergistic for 56,20 , or $10 \%$ of the isolates, respectively. Interestingly synergy of the combination of isavuconazole with tacrolimus was also achieved for the majority of azole-resistant isolates of $A$. fumigatus, and for all $A$. niger isolates with isavuconazole minimal inhibitory concentrations $\geq 8 \mu \mathrm{g} / \mathrm{mL}$. Antagonistic interactions were never observed for any combination tested.
\end{abstract}

Keywords: antifungal combination; in vitro; aspergillosis; Aspergillus; isavuconazole; immunosuppressor; EUCAST

\section{Introduction}

Invasive aspergillosis is a devastating disease in immunocompromised patients associated with a high mortality rate of about 35\% [1]. It mostly affects patients with hematological malignancies, especially those with severe and prolonged neutropenia [2], but is also encountered in solid organ transplant recipients [3-5]. Voriconazole has long been the treatment of choice for invasive aspergillosis [6], and recently isavuconazole expanded the portfolio of first-line treatments [7], but azole-resistance is increasingly reported in Aspergillus fumigatus [8]. In a prospective multicenter international surveillance study, a total of 3788 Aspergillus isolates were screened in 22 centers from 19 countries. Azole-resistant $A$. fumigatus isolates were found in $3.2 \%$ of the cases [9]. The majority of azole-resistant $A$. fumigatus isolates are resistant due to the $\mathrm{TR}_{34} / \mathrm{L} 98 \mathrm{H}$ mutation in the cyp51A gene [10]. The mutation $\mathrm{TR}_{34} / \mathrm{L} 98 \mathrm{H}$ is also found in A. fumigatus isolates cultured from soil and compost. These isolates are cross resistant to azole fungicides and genetically related to clinical azole-resistant aspergilli, showing that the fungicides used for the protection of crops and other plants contribute to the emergence of azole-resistance in A. fumigatus [11]. The high mortality rate among patients with invasive aspergillosis due to multiple triazole resistant $A$. fumigatus isolates, and the possibility of the worldwide 
spread of these resistant isolates by the use of fungicides in agriculture, make azole-resistance in A. fumigatus a major health problem [12]. Isavuconazole is a new board-spectrum azole antifungal drug with excellent activity against most Aspergillus species [13]. Isavuconazole or voriconazole are currently recommended as first-line therapies for pulmonary aspergillosis in Europe [14]. It has been shown that isavuconazole-resistant Aspergillus isolates can be cross-resistant to voriconazole [15,16]. Therefore, combination with antifungals or non-antifungal drugs may be interesting to overcome this resistance. Calcineurin inhibitors (e.g., tacrolimus and cyclosporin A) or inhibitors of the mTOR pathway (e.g., sirolimus) are anti-rejection drugs widely used in organ transplant patients [17], and to prevent graft-versus-host disease in allogeneic stem cell recipients [18], but these immunosuppressive drugs also possess intrinsic antifungal activity against selected fungi, including Candida albicans [19], Cryptococcus neoformans [19,20], A. fumigatus [21], Rhizopus arrhizus [22], and Coccidioides immitis [23]. Calcineurin inhibitors have even exhibited synergy in combination with amphotericin B, posaconazole or isavuconazole against Mucorales [24,25]. Therefore, it is not only of clinical interest, not only if the synergy can also be achieved for Aspergillus species, but also to evaluate calcineurin inhibitors as a potential new antifungal class.

Invasive aspergillosis is a devastating disease in immunocompromised patients associated with a high mortality rate of about $35 \%$ [1]. It mostly affects patients with hematological malignancies, especially those with severe and prolonged neutropenia [2], but is also encountered in solid organ transplant recipients [3-5]. Voriconazole has long been the treatment of choice for invasive aspergillosis [6], and recently isavuconazole expanded the portfolio of first-line treatments [7], but azole-resistance is increasingly reported in Aspergillus fumigatus [8]. In a prospective multicenter international surveillance study, a total of 3788 Aspergillus isolates were screened in 22 centers from 19 countries. Azole-resistant $A$. fumigatus isolates were found in $3.2 \%$ of the cases [9]. The majority of azole-resistant $A$. fumigatus isolates are resistant due to the $\mathrm{TR}_{34} / \mathrm{L} 98 \mathrm{H}$ mutation in the cyp51A gene [10]. The mutation $\mathrm{TR}_{34} / \mathrm{L} 98 \mathrm{H}$ is also found in $A$. fumigatus isolates cultured from soil and compost. These isolates are cross resistant to azole fungicides and genetically related to clinical azole-resistant aspergilli, showing that the fungicides used for the protection of crops and other plants contribute to the emergence of azole-resistance in A. fumigatus [11]. The high mortality rate among patients with invasive aspergillosis due to multiple triazole resistant $A$. fumigatus isolates and the possibility of the worldwide spread of these resistant isolates by the use of fungicides in agriculture make azole-resistance in A. fumigatus a major health problem [12]. Isavuconazole is a new board-spectrum azole antifungal drug with excellent activity against most Aspergillus species [13]. Isavuconazole or voriconazole are currently recommended as first-line therapies for pulmonary aspergillosis in Europe [14]. It has been shown that isavuconazole-resistant Aspergillus isolates can be cross-resistant to voriconazole $[15,16]$. Therefore, combination with antifungals or non-antifungal drugs may be interesting to overcome this resistance. Calcineurin inhibitors (e.g., tacrolimus and cyclosporin A) or inhibitors of the mTOR pathway (e.g., sirolimus) are anti-rejection drugs widely used in organ transplant patients [17] and to prevent graft-versus-host disease in allogeneic stem cell recipients [18], but these immunosuppressive drugs also possess intrinsic antifungal activity against selected fungi, including Candida albicans [19], Cryptococcus neoformans [19,20], A. fumigatus [21], Rhizopus arrhizus [22], and Coccidioides immitis [23]. Calcineurin inhibitors have even exhibited synergy in combination with amphotericin B, posaconazole, or isavuconazole against Mucorales [24,25]. Therefore, it is not only of clinical interest, not only if the synergy can also be achieved for Aspergillus species but also to evaluate calcineurin inhibitors as a potential new antifungal class.

\section{Materials and Methods}

\subsection{Isolates}

A panel of 30 clinical Aspergillus isolates, from the collection of the parasitology/mycology unit of Hôpital Européen Georges-Pompidou (HEGP), belonging to 5 species responsible for human invasive 
aspergillosis was used for the experiments (5 Aspergillus flavus, 10 A. fumigatus, 5 Aspergillus nidulans, 5 Aspergillus niger, and 5 Aspergillus terreus). The isolates of $A$. fumigatus included 5 azole resistant strains (four with TR34/L98H alterations (HEGP-5780, HEGP-4083, HEGP-2659, and HEGP-2664) and one with a G54W mutation (HEGP-4020)). For the other species, isolates were randomly selected to be representative of the species and none of the isolates were known to have specific mechanisms of antifungal resistance. Isolates were subcultured from frozen stocks on Sabouraud dextrose agar slants (Bio-Rad, Feldkirchen, Germany) for 7 days at $35^{\circ} \mathrm{C}$ to ensure purity and viability. The reference strains Candida krusei ATCC 6258 and Candida parapsilosis ATCC 22019 were included in each series of experiments as quality controls.

\subsection{Medium Preparation}

Roswell Park Memorial Institute 1640 (RPMI) medium (with L-glutamine, and pH indicator, but without bicarbonate) (Merck, Darmstadt, Germany) supplemented with dextrose to a final concentration of $2 \%$, buffered with MOPS (Merck) at a final concentration of $0.165 \mathrm{~mol} / \mathrm{L}$, and adjusted to $\mathrm{pH} 7.0$ with $1 \mathrm{M}$ sodium hydroxide was used as a test medium. The medium was prepared at double strength to allow a two-fold dilution. After preparation, the medium was sterilized by vacuum filtration through a $0.22 \mu \mathrm{m}$ pore size filter (Merck).

\subsection{Drugs and Microplate Preparation}

Drug combinations were tested using the EUCAST guidelines for the antifungal susceptibility testing of molds with modifications for a broth microdilution checkerboard procedure, using Nunclon $^{\mathrm{TM}}$ delta surface 96-wells microtiter plates for adherent cells (Thermo Fisher Scientific, Darmstadt, Germany). The included drugs were isavuconazole (Pfizer, Berlin, Germany), tacrolimus (Selleck Chemicals, Munich, Germany), cyclosporin A (Selleck), and sirolimus (Selleck). Stock solutions of drugs were prepared in DMSO. Drug dilutions were performed to four times the final concentrations in double strength RPMI medium. All the combinations were studied on a two-dimensional checkerboard with two-fold dilutions. The final concentrations for isavuconazole were 0.03 to $16 \mu \mathrm{g} / \mathrm{mL}$. The final concentrations for the immunosuppressors were 0.125 to $8 \mu \mathrm{g} / \mathrm{mL}$. Fifty microliters of each concentration were distributed from Rows 1 to 8 for isavuconazole and from Columns 1 to 11 for the immunosuppressive agents. Column 12 was used a as growth control and contained $100 \mu \mathrm{L}$ of double strength RMPI medium with DMSO.

\subsection{Inoculum Preparation and Inoculation of Microplates}

Before inoculum preparation, isolates were subcultured a second time on Sabouraud dextrose agar slants and incubated at $35{ }^{\circ} \mathrm{C}$ under $95 \%$ humidity for 7 days. Spores were transferred to a sterile tube containing water supplemented with $0.1 \%$ of Tween 80 by using a wet cotton swab immersed in sterile water. The suspension was counted in a hemocytometer and adjusted to $2 \times 10^{5}$ conidia $/ \mathrm{mL}$ with sterile water containing $0.1 \%$ of Tween 80 in order to prevent the growth of fungi on the surfaces inside the wells [26]. One hundred microliters of the final inoculum were distributed in each well to inoculate the microdilution plates. The inoculum was further diluted and $100 \mu \mathrm{L}$ were spread twice on Sabouraud dextrose agar plates with a sterile Drigalski spatula. After $24-48 \mathrm{~h}$ of incubation at $35^{\circ} \mathrm{C}$, the colony forming units were counted to ensure the inoculum size and the viability of the conidia. The microplates were incubated at $35{ }^{\circ} \mathrm{C}$ under $95 \%$ humidity, and the minimal inhibitory concentrations (MICs) were determined spectrophotometrically at $48 \mathrm{~h}$ at a wavelength of $530 \mathrm{~nm}$ with the spectrometer MultiSkan FC (Thermo Fisher Scientific). All the experiments were run in duplicate.

\subsection{Interpretation of the Results}

The MICs alone and in combination were determined as the lowest concentrations that caused a complete inhibition as measured by a $90 \%$ of inhibition compared to the control according to spectrophotometric reading. For the calculation of the $\mathrm{MIC}_{50}$, the $\mathrm{MIC}_{90}$, and the geometric mean of 
isavuconazole, the MICs of all three sets were pooled together. For the calculation of the fractional inhibition concentration index (FICI), high off-scale MICs were converted to the next log2 dilutions. The FICI data were interpreted in the following way: FICI $\leq 0.5=$ synergy, FICI $>0.5-4=$ no interaction, and FICI $>4.0=$ antagonism.

\section{Results}

For the combinations of isavuconazole with tacrolimus, cyclosporin A, or sirolimus tested against the 30 Aspergillus isolates by the checkerboard procedure, the MICs of the drugs alone, the MICs in combination, and the corresponding interaction for the lowest FIC indices are presented in Table 1. A summary of the results for all the combinations is presented in Table 2. The thirty isolates exhibited MICs for isavuconazole alone ranging from 0.25 to $16 \mu \mathrm{g} / \mathrm{mL}$ (Table 1) with a $\mathrm{MIC}_{50}, \mathrm{MIC}_{90}$, and geometric mean MIC of 1,16 , and $2.06 \mu \mathrm{g} / \mathrm{mL}$, respectively. Isavuconazole MICs for A. flavus, A. fumigatus, A. nidulans, A. niger, and A. terreus ranged from 2 to 4,1 to $16,0.25$ to $0.5,4$ to 16 , and 0.5 to $1 \mu \mathrm{g} / \mathrm{ml}$, respectively. Between experiments, the isavuconazole MICs were within $+/-1 \log _{2}$ dilutions in $100 \%$ of the cases. Immunosuppressive drugs alone did not exhibit in vitro activity, except for four isolates. The tacrolimus MICs were $>8 \mu \mathrm{g} / \mathrm{mL}$, except for one A. niger (HEGP-6917) and two A. terreus (HEGP-6398, HEGP-6625) isolates. The cyclosporin A MICs were $>8 \mu \mathrm{g} / \mathrm{mL}$, except for one A. niger (HEGP-6917) and two A. terreus (HEGP-5599, HEGP-6398) isolates. For sirolimus, all the isolates exhibited MICs $>8 \mu \mathrm{g} / \mathrm{mL}$. The interactions of isavuconazole with tacrolimus were synergistic for $50 \%$ of the isolates with FICIs ranging from 0.015 to 0.5 . A. fumigatus isolates with mechanisms of resistance to azoles showed synergy for $60 \%$ of the isolates (three out of five isolates) and $100 \%$ synergy for all the $A$. niger isolates with isavuconazole MICs $\geq 8 \mu \mathrm{g} / \mathrm{mL}$ (four out of four isolates). For the other A. niger isolate (HEGP-6917) and two A. terreus isolates (HEGP-6398 and HEGP-6625), synergy was not detectable with the tacrolimus concentrations used on the microplates, because the MICs of tacrolimus alone were too low $(0.25 \mu \mathrm{g} / \mathrm{mL})$. Therefore, these isolates were excluded from the calculation of percentages of interaction of Table 2. For the combination of isavuconazole with cyclosporin A synergistic interactions were observed for $20 \%$ of the isolates. (FICI ranging from 0.13 to 0.5). Synergy was seen for $80 \%$ of the A. niger isolates; similar to what was observed for the combination with tacrolimus, synergy was obtained despite high MICs to isavuconazole of $\geq 8 \mu \mathrm{g} / \mathrm{mL}$. For $A$. terreus synergy was seen for $40 \%$ of the isolates. For the combination of isavuconazole with sirolimus synergy was obtained for $10 \%$ of the isolates (FICI ranging from 0.19 to 0.38 ), comprising two A. flavus isolates and one $A$. terreus isolate. Antagonistic interactions were never observed for any combination tested. 
Table 1. Interaction of isavuconazole with tacrolimus, cyclosporin A, or sirolimus against Aspergillus species.

\begin{tabular}{|c|c|c|c|c|c|c|c|c|c|c|c|c|c|c|c|c|}
\hline \multirow{2}{*}{ Species } & \multirow{2}{*}{ Collection Number } & \multicolumn{3}{|c|}{ MIC $(\mu \mathrm{g} / \mathrm{mL})$} & \multirow[b]{2}{*}{ FICI } & \multirow[b]{2}{*}{ INTPN } & \multicolumn{3}{|c|}{ MIC $(\mu \mathrm{g} / \mathrm{mL})$} & \multirow[b]{2}{*}{ FICI } & \multirow[b]{2}{*}{ INTPN } & \multicolumn{3}{|c|}{ MIC $(\mu \mathrm{g} / \mathrm{mL})$} & \multirow[b]{2}{*}{ FICI } & \multirow[b]{2}{*}{ INTPN } \\
\hline & & IVZ & TAC $^{d}$ & IVZ/TAC & & & IVZ & $\mathrm{CYA}^{\mathrm{d}}$ & IVZ/CYA & & & IVZ & SLM $^{d}$ & IVZ/SLM & & \\
\hline A. flavus & HEGP-6097 & 4 & 16 & $2 / 1$ & 0.5625 & IND & 4 & 16 & $4 / 0.12$ & 1.0078 & IND & 4 & 16 & $1 / 2$ & 0.375 & SYN \\
\hline A. flavus & HEGP-5899 & 4 & 16 & $0.5 / 8$ & 0.625 & IND & 4 & 16 & $4 / 0.12$ & 1.0078 & IND & 4 & 16 & $2 / 0.25$ & 0.5156 & IND \\
\hline A. flavus & HEGP-4536 & 2 & 16 & $0.06 / 4$ & 0.2813 & SYN & 2 & 16 & $2 / 0.12$ & 1.0078 & IND & 2 & 16 & $1 / 1$ & 0.5625 & IND \\
\hline A. flavus & HEGP-4251 & 2 & 16 & $0.03 / 4$ & 0.2656 & SYN & 4 & 16 & $2 / 0.12$ & 0.5078 & IND & 2 & 16 & $0.5 / 1$ & 0.3125 & SYN \\
\hline A. flavus & HEGP-4114 & 2 & 16 & $0.5 / 4$ & 0.5 & SYN & 2 & 16 & $2 / 0.25$ & 1.0156 & IND & 2 & 16 & $1 / 1$ & 0.5625 & IND \\
\hline A. fumigatus & HEGP- $5780^{\mathrm{b}}$ & 16 & 16 & $8 / 1$ & 0.5625 & IND & 8 & 16 & $16 / 0.12$ & 2.0078 & IND & 16 & 16 & $8 / 2$ & 0.625 & IND \\
\hline A. fumigatus & HEGP- $4020^{c}$ & 1 & 16 & $0.5 / 2$ & 0.625 & IND & 2 & 16 & $1 / 0.12$ & 0.5078 & IND & 1 & 16 & $0.5 / 2$ & 0.625 & IND \\
\hline A. fumigatus & HEGP-4083 b & 16 & 16 & $4 / 4$ & 0.5 & SYN & 16 & 16 & $16 / 0.12$ & 1.0078 & IND & 16 & 16 & $16 / 0.12$ & 1.0078 & IND \\
\hline A. fumigatus & HEGP-2659 b & 16 & 16 & $4 / 2$ & 0.375 & SYN & 8 & 16 & $16 / 0.12$ & 2.0078 & IND & 16 & 16 & $8 / 8$ & 1.0 & IND \\
\hline A. fumigatus & HEGP-2664 b & 8 & 16 & $2 / 4$ & 0.5 & SYN & 8 & 16 & $8 / 0.12$ & 1.0078 & IND & 16 & 16 & $8 / 0.12$ & 0.5078 & IND \\
\hline A. fumigatus & HEGP-R117 & 1 & 16 & $0.25 / 2$ & 0.375 & SYN & 1 & 16 & $1 / 0.12$ & 1.0078 & IND & 1 & 16 & $1 / 0.12$ & 1.0078 & IND \\
\hline A. fumigatus & HEGP-R279 & 1 & 16 & $0.5 / 4$ & 0.75 & IND & 1 & 16 & $1 / 0.12$ & 1.0078 & IND & 1 & 16 & $0.5 / 0.12$ & 0.75 & IND \\
\hline A. fumigatus & HEGP-R285 & 1 & 16 & $0.5 / 1$ & 0.5625 & IND & 1 & 16 & $1 / 0.12$ & 1.0078 & IND & 1 & 16 & $1 / 0.12$ & 1.0078 & IND \\
\hline A. fumigatus & HEGP-R290 & 2 & 16 & $1 / 0.5$ & 0.5313 & IND & 1 & 16 & $1 / 8$ & 1.5 & IND & 2 & 16 & $1 / 8$ & 1.0 & IND \\
\hline A. fumigatus & HEGP-R291 & 1 & 16 & $0.5 / 2$ & 0.625 & IND & 1 & 16 & $1 / 0.12$ & 1.0078 & IND & 1 & 16 & $1 / 0.12$ & 1.0078 & IND \\
\hline A. nidulans & HEGP-5711 & 0.25 & 16 & $0.12 / 4$ & 0.75 & IND & 0.5 & 16 & $0.25 / 2$ & 0.625 & IND & 0.5 & 16 & $0.5 / 0.12$ & 1.0078 & IND \\
\hline A. nidulans & HEGP-6169 & 0.5 & 16 & $0.12 / 4$ & 0.5 & SYN & 0.5 & 16 & $0.25 / 2$ & 0.625 & IND & 0.5 & 16 & $0.5 / 0.12$ & 1.0078 & IND \\
\hline A. nidulans & HEGP-5492 & 0.5 & 16 & $0.25 / 1$ & 0.5625 & IND & 0.5 & 16 & $0.25 / 2$ & 0.625 & IND & 0.5 & 16 & $0.5 / 0.12$ & 1.0078 & IND \\
\hline A. nidulans & HEGP-5521 & 0.5 & 16 & $0.25 / 0.5$ & 0.5313 & IND & 0.5 & 16 & $0.25 / 4$ & 0.75 & IND & 0.5 & 16 & $0.5 / 0.12$ & 1.0078 & IND \\
\hline A. nidulans & HEGP-5329 & 0.5 & 16 & $0.25 / 1$ & 0.5625 & IND & 0.5 & 16 & $0.25 / 2$ & 0.625 & IND & 0.5 & 16 & $0.5 / 0.12$ & 1.0078 & IND \\
\hline A. niger & HEGP-6071 & 16 & 16 & $0.25 / 0.25$ & 0.0313 & SYN & 16 & 16 & $0.25 / 2$ & 0.1406 & SYN & 16 & 16 & $16 / 0.12$ & 1.0078 & IND \\
\hline A. niger & HEGP-6217 & 8 & 16 & $0.06 / 0.25$ & 0.0234 & SYN & 8 & 16 & $1 / 2$ & 0.25 & SYN & 8 & 16 & $4 / 2$ & 0.625 & IND \\
\hline A. niger & HEGP-6475 & 16 & 16 & $0.06 / 0.25$ & 0.0195 & SYN & 16 & 16 & $0.12 / 2$ & 0.1328 & SYN & 16 & 16 & $8 / 0.12$ & 0.5078 & IND \\
\hline A. niger & HEGP-6562 & 16 & 16 & $0.12 / 0.12$ & 0.0156 & SYN & 16 & 16 & $0.25 / 2$ & 0.1406 & SYN & 16 & 16 & $8 / 0.12$ & 0.5078 & IND \\
\hline A. niger & HEGP-6917 & 4 & 0.25 & $0.03 / 0.25$ & 1.0078 & $-a$ & 4 & 4 & $2 / 2$ & 1.0 & IND & 8 & 16 & $4 / 0.12$ & 0.5078 & IND \\
\hline A. terreus & HEGP-6625 & 0.5 & 0.25 & $0.12 / 0.12$ & 0.75 & $-{ }^{a}$ & 1 & 16 & $0.06 / 2$ & 0.1875 & SYN & 1 & 16 & $0.5 / 0.12$ & 0.5078 & IND \\
\hline A. terreus & HEGP-6055 & 1 & 16 & $0.25 / 2$ & 0.375 & SYN & 1 & 16 & $0.25 / 4$ & 0.5 & SYN & 1 & 16 & $0.5 / 8$ & 1.0 & IND \\
\hline A. terreus & HEGP-5599 & 0.5 & 16 & $0.03 / 0.12$ & 0.0703 & SYN & 0.5 & 1 & $0.25 / 0.5$ & 1.0 & IND & 0.25 & 16 & $0.12 / 8$ & 1.0 & IND \\
\hline A. terreus & HEGP-5169 & 0.5 & 16 & $0.12 / 0.5$ & 0.2813 & SYN & 0.5 & 16 & $0.25 / 2$ & 0.625 & IND & 0.5 & 16 & $0.25 / 0.5$ & 0.5313 & IND \\
\hline A. terreus & HEGP-6398 & 0.5 & 0.25 & $0.03 / 0.12$ & 0.5625 & $-a$ & 0.5 & 1 & $0.06 / 0.5$ & 0.625 & IND & 0.5 & 16 & $0.06 / 1$ & 0.1875 & SYN \\
\hline
\end{tabular}

MIC, minimal inhibitory concentration; FICI, fractional inhibitory concentration index; INTPN, interpretation; SYN, synergy (FICI $\leq 0.5)$; IND, no interaction $(0.5<$ FICI $\leq 4)$, ANT antagonism $\left(\mathrm{FICI}>4\right.$ ). IVZ, isavuconazole; TAC, tacrolimus; CYA, cyclosporin A; SLM, sirolimus; HEGP, Hôpital Européen Georges-Pompidou; ${ }^{a}$ differentiation between synergy
and no interaction not possible, as MICs of immune suppressors alone were too low (isolate excluded); ${ }^{\mathrm{b}}$ isolate with TR34/L98H alteration; ${ }^{\mathrm{c}}$ isolate with G54W mutation; ${ }^{\mathrm{d}}$ MICs for immunosuppressors reported as $16 \mu \mathrm{g} / \mathrm{mL}$ were $>8 \mu \mathrm{g} / \mathrm{mL}$. 
Table 2. Summary of interactions of isavuconazole with tacrolimus, cyclosporin A, or sirolimus against Aspergillus species interpreted based on the fractional inhibitory concentration index.

\begin{tabular}{cccccccccc}
\hline & \multicolumn{4}{c}{$\%$ of Isolates with the Following Interaction ${ }^{\text {a }}$} \\
\cline { 2 - 10 } Species (Number of Isolates) & \multicolumn{3}{c}{ Synergy } & \multicolumn{3}{c}{ No Interaction } & \multicolumn{3}{c}{ Antagonism } \\
\cline { 2 - 10 } & TAC & CYA & SLM & TAC & CYA & SLM & TAC & CYA & SLM \\
\hline A. flavus (5) & 60 & 0 & 40 & 40 & 100 & 60 & 0 & 0 & 0 \\
A. fumigatus (10) & 40 & 0 & 0 & 60 & 100 & 100 & 0 & 0 & 0 \\
A. nidulans (5) & 20 & 0 & 0 & 80 & 100 & 100 & 0 & 0 & 0 \\
A. niger (5) & $100^{\mathrm{b}}$ & 80 & 0 & 0 & 20 & 100 & 0 & 0 & 0 \\
A. terreus (5) & $100^{\mathrm{b}}$ & 40 & 20 & 0 & 60 & 80 & 0 & 0 & 0 \\
All (30) & $56^{\mathrm{b}}$ & 20 & 10 & $44^{\mathrm{b}}$ & 80 & 90 & 0 & 0 & 0 \\
\hline
\end{tabular}

${ }^{a}$ all immunosuppressive agents were combined with isavuconazole; TAC, tacrolimus; CYA, cyclosporin A; SLM, sirolimus; ${ }^{\mathrm{b}}$ for the calculation of percentages 1 A. niger and 2 A. terreus isolates were excluded, as differentiation between synergy and no interaction was not possible with the concentrations of tacrolimus chosen on the plates.

\section{Discussion}

Immunosuppressive drugs such as calcineurin or mTOR pathway inhibitors are used as anti-rejection drugs in organ transplant, and allogeneic stem cell recipients. The calcineurin inhibitors lead to a reduced activity of cytokine genes, finally leading to the reduced proliferation of $\mathrm{T}$ lymphocytes $[27,28]$. Inhibitors of the mTOR pathway lead to an arrest of the cell-cycle in the late G1/S phase of $\mathrm{T}$ and B lymphocytes, preventing proliferation [29]. Beside these anti-proliferative properties, the drugs also possess intrinsic antifungal activity against yeasts $[19,20]$ and filamentous $[21,22]$ and dimorphic fungi [23]. Here, we found that immunosuppressors had no antifungal activity alone, except for four isolates ( $A$. niger and $A$. terreus). It would be interesting to test a higher number of isolates to know if this is species or strain specific.

In vitro synergy between antifungals and immune suppressive drugs has been found for yeasts [30-36], and filamentous fungi such as the Mucorales [24,25,37,38], and Aspergillus species [39,40]. Nevertheless, in vitro indifference [21,41] and even antagonism has been reported for the combinations of voriconazole with tacrolimus or cyclosporin A against four A. fumigatus isolates and one A. fumigatus isolate, respectively [41]. Indifference has also been reported for the combinations of posaconazole or itraconazole with tacrolimus against Aspergillus biofilms [39]. One of the two studies demonstrating in vitro synergy between antifungals and immunosuppressors evaluated the combination of caspofungin in combination with tacrolimus, cyclosporin $\mathrm{A}$, sirolimus, or other calcineurin inhibitors by a disc diffusion assay against 13 Aspergillus, mostly A. fumigatus isolates. The inhibition zones for tacrolimus or sirolimus in combination with caspofungin were significantly larger compared to those for caspofungin alone for the $10 \mathrm{~A}$. fumigatus isolates at $48 \mathrm{~h}$. The same results were seen for one $A$. terreus isolate, but for neither the other $A$. terreus isolate nor for the A. flavus isolate [40]. In this study, the immunosuppressive drugs showed poor in vitro activity when tested alone, in contrast with previous reports [21,42]. This could be related to differences in the technique used, and particularly, to the more stringent endpoint ( $90 \%$ inhibition) used in our study. The isavuconazole MICs of the tested Aspergillus isolates determined by ECUAST methodology were in the same range as previously reported [43]. The combination of isavuconazole with tacrolimus exhibited a synergistic effect (56\% of the isolates) against Aspergillus species, including $60 \%$ of A. fumigatus isolates with mechanisms of resistance to azoles and all $A$. niger isolates with isavuconazole MICs $\geq 8 \mu \mathrm{g} / \mathrm{mL}$. As tacrolimus is a known inhibitor of efflux pumps [44], it could be speculated that synergy may be more frequent in azole-resistant strains with an overexpression of efflux pumps. Therefore, it could be of interest to determine the level of expression of efflux pumps in our isolates. The presence of a known mechanism of resistance in our azole-resistant $A$. fumigatus strains (with cyp51A mutations and promotor alteration) did not rule out the possibility of higher efflux in these isolates. Our results are in accordance with a study that evaluated the interaction of voriconazole with tacrolimus against Aspergillus biofilms. The combination was tested against twenty Aspergillus biofilms and ten 
A. fumigatus, eight $A$. flavus, and two A. terreus isolates. Overall synergy was achieved for $60 \%$ of the tested isolates [39]. Why combinations of tacrolimus with isavuconazole or voriconazole exhibit synergy and combinations of tacrolimus with posaconazole or itraconazole exhibit only indifference remains unknown. It is possible that the different interactions are related to the steric structures of the molecules. Synergistic interactions between tacrolimus and isavuconazole may be of particular interest when tacrolimus analogs with lower immunosuppressive activity become available $[45,46]$. The combination of isavuconazole with cyclosporin A led to less synergistic interactions $(20 \%$ of the isolates) than the combination with tacrolimus. The combination of isavuconazole with sirolimus was synergistic for $10 \%$ of the isolates. Similar results have already been seen for voriconazole in combination with the three immunosuppressors used in this study against A. fumigatus. All the interactions were indifferent [21].

It has to be pointed out that the concentrations for which synergistic interactions were achieved for tacrolimus, cyclosporin, and sirolimus in this study, were above the peak drug levels in clinical practice of $0.025 \mu \mathrm{g} / \mathrm{mL}, 1.2 \mu \mathrm{g} / \mathrm{mL}$, and $0.02 \mu \mathrm{g} / \mathrm{mL}$, respectively [47,48]. Nevertheless, analyses of the fractional inhibitory concentration indices are limited to the exploration of the MIC endpoints and the tested concentrations on the microplates. From these data, it cannot be excluded that synergistic interactions could be present at lower concentrations than those tested on the microplate. In another study, tacrolimus was tested by the same technique used in this study at subtherapeutic concentrations of $0.04-25 \mu \mathrm{g} / \mathrm{mL}$ in combination with amphotericin B or fluconazole. Synergy was obtained for 90 and $82 \%$ of the isolates, respectively. In the same study, the outcomes in solid organ transplant recipients with cryptococcosis receiving tacrolimus long-term therapy and amphotericin B or fluconazole were significantly better, regarding survival, than those of patients receiving only amphotericin B or fluconazole therapy without tacrolimus [49].

In summary, immunosuppressors can enhance the in vitro activity of isavuconazole against Aspergillus species. The best activity was seen for the combination of tacrolimus with isavuconazole. The combination was active against all the tested species, including $A$. fumigatus isolates with resistance to azoles and $A$. niger isolates with high isavuconazole MICs. The combination of cyclosporin with isavuconazole was active against all the $A$. niger isolates with high isavuconazole MICs. These in vitro results warrant further animal experiments.

Author Contributions: P.S. performed the experiments and created the first draft of the manuscript. P.S. and E.D. performed the analysis of the results. E.D. and P.S. contributed to the revisions. Both authors drafted the final article, approved the publication, and agreed to be accountable for all aspects of the work. All authors have read and agreed to the published version of the manuscript.

Acknowledgments: The authors are grateful to Pfizer for providing the drug isavuconazole for this research project.

Conflicts of Interest: Patrick Schwarz has received research grants from Basilea Pharmaceutica, Gilead and Pfizer and received a travel grants from Gilead and Pfizer. During the past 5 years, Eric Dannaoui has received research grants from MSD and Gilead; travel grants from Gilead, MSD, Pfizer, and Astellas, and speaker's fee from Gilead, MSD, and Astellas.

Congress Presentations: The results of this work (abstract number P059) were presented at the 9th Trends in Medical Mycology, 11th-14th October 2019 in Nice, France.

\section{References}

1. Steinbach, W.J.; Marr, K.A.; Anaissie, E.J.; Azie, N.; Quan, S.P.; Meier-Kriesche, H.U.; Apewokin, S.; Horn, D.L. Clinical epidemiology of 960 patients with invasive aspergillosis from the path alliance registry. J. Infect. 2012, 65, 453-464. [CrossRef]

2. Segal, B.H. Aspergillosis. N. Engl. J. Med. 2009, 360, 1870-1884. [CrossRef]

3. Heylen, L.; Maertens, J.; Naesens, M.; Van Wijngaerden, E.; Lagrou, K.; Bammens, B.; Claes, K.; Evenepoel, P.; Meijers, B.; Kuypers, D.; et al. Invasive aspergillosis after kidney transplant: Case-control study. Clin. Infect. Dis. 2015, 60, 1505-1511. [CrossRef] 
4. Iversen, M.; Burton, C.M.; Vand, S.; Skovfoged, L.; Carlsen, J.; Milman, N.; Andersen, C.B.; Rasmussen, M.; Tvede, M. Aspergillus infection in lung transplant patients: Incidence and prognosis. Eur. J. Clin. Microbiol. Infect. Dis. 2007, 26, 879-886. [CrossRef]

5. Rosenhagen, M.; Feldhues, R.; Schmidt, J.; Hoppe-Tichy, T.; Geiss, H.K. A risk profile for invasive aspergillosis in liver transplant recipients. Infection 2009, 37, 313-319. [CrossRef]

6. Herbrecht, R.; Denning, D.W.; Patterson, T.F.; Bennett, J.E.; Greene, R.E.; Oestmann, J.W.; Kern, W.V.; Marr, K.A.; Ribaud, P.; Lortholary, O.; et al. Voriconazole versus amphotericin b for primary therapy of invasive aspergillosis. N. Engl. J. Med. 2002, 347, 408-415. [CrossRef]

7. Maertens, J.A.; Raad, I.I.; Marr, K.A.; Patterson, T.F.; Kontoyiannis, D.P.; Cornely, O.A.; Bow, E.J.; Rahav, G.; Neofytos, D.; Aoun, M.; et al. Isavuconazole versus voriconazole for primary treatment of invasive mould disease caused by Aspergillus and other filamentous fungi (SECURE): A phase 3, randomised-controlled, non-inferiority trial. Lancet 2016, 387, 760-769. [CrossRef]

8. Chowdhary, A.; Kathuria, S.; Xu, J.; Meis, J.F. Emergence of azole-resistant Aspergillus fumigatus strains due to agricultural azole use creates an increasing threat to human health. PLoS Pathog. 2013, 9, e1003633. [CrossRef]

9. van der Linden, J.W.; Arendrup, M.C.; Warris, A.; Lagrou, K.; Pelloux, H.; Hauser, P.M.; Chryssanthou, E.; Mellado, E.; Kidd, S.E.; Tortorano, A.M.; et al. Prospective multicenter international surveillance of azole resistance in Aspergillus fumigatus. Emerg. Infect. Dis. 2015, 21, 1041-1044. [CrossRef]

10. Lockhart, S.R.; Frade, J.P.; Etienne, K.A.; Pfaller, M.A.; Diekema, D.J.; Balajee, S.A. Azole resistance in Aspergillus fumigatus isolates from the artemis global surveillance study is primarily due to the TR/L98H mutation in the cyp51A gene. Antimicrob. Agents Chemother. 2011, 55, 4465-4468. [CrossRef]

11. Verweij, P.E.; Snelders, E.; Kema, G.H.; Mellado, E.; Melchers, W.J. Azole resistance in Aspergillus fumigatus: A side-effect of environmental fungicide use? Lancet Infect. Dis. 2009, 9, 789-795. [CrossRef]

12. Verweij, P.E.; Chowdhary, A.; Melchers, W.J.; Meis, J.F. Azole resistance in Aspergillus fumigatus: Can we retain the clinical use of mold-active antifungal azoles? Clin. Infect. Dis. 2016, 62, 362-368. [CrossRef] [PubMed]

13. Messer, S.A.; Carvalhaes, C.G.; Castanheira, M.; Pfaller, M.A. In vitro activity of isavuconazole versus opportunistic filamentous fungal pathogens from the SENTRY antifungal surveillance program, 2017-2018. Diagn. Microbiol. Infect. Dis. 2020, 97, 115007. [CrossRef]

14. Ullmann, A.J.; Aguado, J.M.; Arikan-Akdagli, S.; Denning, D.W.; Groll, A.H.; Lagrou, K.; Lass-Florl, C.; Lewis, R.E.; Munoz, P.; Verweij, P.E.; et al. Diagnosis and management of Aspergillus diseases: Executive summary of the 2017 ESCMID-ECMM-ERS guideline. Clin. Microbiol. Infect. 2018, 24 (Suppl. 1), e1-e38. [CrossRef] [PubMed]

15. Chowdhary, A.; Sharma, C.; van den Boom, M.; Yntema, J.B.; Hagen, F.; Verweij, P.E.; Meis, J.F. Multi-azole-resistant Aspergillus fumigatus in the environment in tanzania. J. Antimicrob. Chemother. 2014, 69, 2979-2983. [CrossRef] [PubMed]

16. Gregson, L.; Goodwin, J.; Johnson, A.; McEntee, L.; Moore, C.B.; Richardson, M.; Hope, W.W.; Howard, S.J. In vitro susceptibility of Aspergillus fumigatus to isavuconazole: Correlation with itraconazole, voriconazole, and posaconazole. Antimicrob. Agents Chemother. 2013, 57, 5778-5780. [CrossRef] [PubMed]

17. Gummert, J.F.; Ikonen, T.; Morris, R.E. Newer immunosuppressive drugs: A review. J. Am. Soc. Nephrol. 1999, 10, 1366-1380.

18. Choi, S.W.; Reddy, P. Current and emerging strategies for the prevention of graft-versus-host disease. Nat. Rev. Clin. Oncol. 2014, 11, 536-547. [CrossRef]

19. Cruz, M.C.; Goldstein, A.L.; Blankenship, J.; Del Poeta, M.; Perfect, J.R.; McCusker, J.H.; Bennani, Y.L.; Cardenas, M.E.; Heitman, J. Rapamycin and less immunosuppressive analogs are toxic to Candida albicans and Cryptococcus neoformans via FKBP12-dependent inhibition of TOR. Antimicrob. Agents Chemother. 2001, 45, 3162-3170. [CrossRef]

20. Cruz, M.C.; Del Poeta, M.; Wang, P.; Wenger, R.; Zenke, G.; Quesniaux, V.F.; Movva, N.R.; Perfect, J.R.; Cardenas, M.E.; Heitman, J. Immunosuppressive and nonimmunosuppressive cyclosporine analogs are toxic to the opportunistic fungal pathogen Cryptococcus neoformans via cyclophilin-dependent inhibition of calcineurin. Antimicrob. Agents Chemother. 2000, 44, 143-149. [CrossRef] 
21. Steinbach, W.J.; Singh, N.; Miller, J.L.; Benjamin, D.K., Jr.; Schell, W.A.; Heitman, J.; Perfect, J.R. In vitro interactions between antifungals and immunosuppressants against Aspergillus fumigatus isolates from transplant and nontransplant patients. Antimicrob. Agents Chemother. 2004, 48, 4922-4925. [CrossRef]

22. Lewis, R.E.; Ben-Ami, R.; Best, L.; Albert, N.; Walsh, T.J.; Kontoyiannis, D.P. Tacrolimus enhances the potency of posaconazole against Rhizopus oryzae in vitro and in an experimental model of mucormycosis. J. Infect. Dis. 2013, 207, 834-841. [CrossRef] [PubMed]

23. Kirkland, T.N.; Fierer, J. Cyclosporin a inhibits Coccidioides immitis in vitro and invivo. Antimicrob. Agents Chemother. 1983, 24, 921-924. [CrossRef] [PubMed]

24. Dannaoui, E.; Schwarz, P.; Lortholary, O. In vitro interactions between antifungals and immunosuppressive drugs against zygomycetes. Antimicrob. Agents Chemother. 2009, 53, 3549-3551. [CrossRef]

25. Schwarz, P.; Schwarz, P.V.; Felske-Zech, H.; Dannaoui, E. In vitro interactions between isavuconazole and tacrolimus, cyclosporin a or sirolimus against Mucorales. J. Antimicrob. Chemother. 2019, 74, $1921-1927$. [CrossRef] [PubMed]

26. Meletiadis, J.; Meis, J.F.; Mouton, J.W.; Verweij, P.E. Analysis of growth characteristics of filamentous fungi in different nutrient media. J. Clin. Microbiol. 2001, 39, 478-484. [CrossRef]

27. Schreiber, S.L.; Crabtree, G.R. The mechanism of action of cyclosporin a and FK506. Immunol. Today 1992, 13, 136-142. [CrossRef]

28. Wiederrecht, G.; Lam, E.; Hung, S.; Martin, M.; Sigal, N. The mechanism of action of FK-506 and cyclosporin A. Ann. N. Y. Acad. Sci. 1993, 696, 9-19. [CrossRef]

29. Dumont, F.J.; Su, Q. Mechanism of action of the immunosuppressant rapamycin. Life Sci 1996, 58, 373-395. [CrossRef]

30. Del Poeta, M.; Cruz, M.C.; Cardenas, M.E.; Perfect, J.R.; Heitman, J. Synergistic antifungal activities of bafilomycin a1, fluconazole, and the pneumocandin MK-0991/caspofungin acetate (L-743,873) with calcineurin inhibitors FK506 and L-685,818 against Cryptococcus neoformans. Antimicrob. Agents Chemother. 2000, 44, 739-746. [CrossRef]

31. Li, Y.; Sun, S.; Guo, Q.; Ma, L.; Shi, C.; Su, L.; Li, H. In vitro interaction between azoles and cyclosporin A against clinical isolates of Candida albicans determined by the chequerboard method and time-kill curves. J. Antimicrob. Chemother. 2008, 61, 577-585. [CrossRef]

32. Maesaki, S.; Marichal, P.; Hossain, M.A.; Sanglard, D.; Vanden Bossche, H.; Kohno, S. Synergic effects of tacrolimus and azole antifungal agents against azole-resistant Candida albicans strains. J. Antimicrob. Chemother. 1998, 42, 747-753. [CrossRef] [PubMed]

33. Marchetti, O.; Moreillon, P.; Glauser, M.P.; Bille, J.; Sanglard, D. Potent synergism of the combination of fluconazole and cyclosporine in candida albicans. Antimicrob. Agents Chemother. 2000, 44, 2373-2381. [CrossRef] [PubMed]

34. Onyewu, C.; Blankenship, J.R.; Del Poeta, M.; Heitman, J. Ergosterol biosynthesis inhibitors become fungicidal when combined with calcineurin inhibitors against Candida albicans, Candida glabrata, and Candida krusei. Antimicrob. Agents Chemother. 2003, 47, 956-964. [CrossRef] [PubMed]

35. Singh, N.; Alexander, B.D.; Lortholary, O.; Dromer, F.; Gupta, K.L.; John, G.T.; del Busto, R.; Klintmalm, G.B.; Somani, J.; Lyon, G.M.; et al. Cryptococcus neoformans in organ transplant recipients: Impact of calcineurin-inhibitor agents on mortality. J. Infect. Dis. 2007, 195, 756-764. [CrossRef]

36. Sun, S.; Li, Y.; Guo, Q.; Shi, C.; Yu, J.; Ma, L. In vitro interactions between tacrolimus and azoles against Candida albicans determined by different methods. Antimicrob. Agents Chemother. 2008, 52, 409-417. [CrossRef]

37. Narreddy, S.; Manavathu, E.; Chandrasekar, P.H.; Alangaden, G.J.; Revankar, S.G. In vitro interaction of posaconazole with calcineurin inhibitors and sirolimus against zygomycetes. J. Antimicrob. Chemother. 2010, 65, 701-703. [CrossRef]

38. Schwarz, P.; Cornely, O.A.; Dannaoui, E. Antifungal combinations in mucorales: A microbiological perspective. Mycoses 2019, 62, 746-760. [CrossRef]

39. Gao, L.; Sun, Y. In vitro interactions of antifungal agents and tacrolimus against Aspergillus biofilms. Antimicrob. Agents Chemother. 2015, 59, 7097-7099. [CrossRef]

40. Kontoyiannis, D.P.; Lewis, R.E.; Osherov, N.; Albert, N.D.; May, G.S. Combination of caspofungin with inhibitors of the calcineurin pathway attenuates growth in vitro in Aspergillus species. J. Antimicrob. Chemother. 2003, 51, 313-316. [CrossRef] 
41. Steinbach, W.J.; Schell, W.A.; Blankenship, J.R.; Onyewu, C.; Heitman, J.; Perfect, J.R. In vitro interactions between antifungals and immunosuppressants against Aspergillus fumigatus. Antimicrob. Agents Chemother. 2004, 48, 1664-1669. [CrossRef] [PubMed]

42. Lamoth, F.; Alexander, B.D.; Juvvadi, P.R.; Steinbach, W.J. Antifungal activity of compounds targeting the Hsp90-calcineurin pathway against various mould species. J. Antimicrob. Chemother. 2015, 70, 1408-1411. [CrossRef] [PubMed]

43. Howard, S.J.; Lass-Florl, C.; Cuenca-Estrella, M.; Gomez-Lopez, A.; Arendrup, M.C. Determination of isavuconazole susceptibility of Aspergillus and Candida species by the EUCAST method. Antimicrob. Agents Chemother. 2013, 57, 5426-5431. [CrossRef]

44. Holmes, A.R.; Cardno, T.S.; Strouse, J.J.; Ivnitski-Steele, I.; Keniya, M.V.; Lackovic, K.; Monk, B.C.; Sklar, L.A.; Cannon, R.D. Targeting efflux pumps to overcome antifungal drug resistance. Future Med. Chem. 2016, 8, 1485-1501. [CrossRef]

45. Juvvadi, P.R.; Fox, D., 3rd; Bobay, B.G.; Hoy, M.J.; Gobeil, S.M.C.; Venters, R.A.; Chang, Z.; Lin, J.J.; Averette, A.F.; Cole, D.C.; et al. Harnessing calcineurin-FK506-FKBP12 crystal structures from invasive fungal pathogens to develop antifungal agents. Nat. Commun. 2019, 10, 4275. [CrossRef]

46. Lee, Y.; Lee, K.T.; Lee, S.J.; Beom, J.Y.; Hwangbo, A.; Jung, J.A.; Song, M.C.; Yoo, Y.J.; Kang, S.H.; Averette, A.F.; et al. In vitro and in vivo assessment of FK506 analogs as novel antifungal drug candidates. Antimicrob. Agents Chemother. 2018, 62, e01627-18. [CrossRef] [PubMed]

47. Falck, P.; Vethe, N.T.; Asberg, A.; Midtvedt, K.; Bergan, S.; Reubsaet, J.L.; Holdaas, H. Cinacalcet's effect on the pharmacokinetics of tacrolimus, cyclosporine and mycophenolate in renal transplant recipients. Nephrol. Dial. Transplant. 2008, 23, 1048-1053. [CrossRef] [PubMed]

48. Mahalati, K.; Kahan, B.D. Clinical pharmacokinetics of sirolimus. Clin. Pharmacokinet. 2001, 40, 573-585. [CrossRef] [PubMed]

49. Kontoyiannis, D.P.; Lewis, R.E.; Alexander, B.D.; Lortholary, O.; Dromer, F.; Gupta, K.L.; John, G.T.; Del Busto, R.; Klintmalm, G.B.; Somani, J.; et al. Calcineurin inhibitor agents interact synergistically with antifungal agents in vitro against Cryptococcus neoformans isolates: Correlation with outcome in solid organ transplant recipients with cryptococcosis. Antimicrob. Agents Chemother. 2008, 52, 735-738. [CrossRef] 